\title{
Video review
}

\section{Belong Out There}

'I Belong Out There', produced by Irene $M$. Ward and Associates and enacted by Clyde Yoder, is a 'working' video. It does refer to work and coworkers, however, it is not about work. It is about:

- Individuals with disabilities becoming involved in social and recreational activities.

- Self-advocacy.

- Building community connections.

- Making friends.

- Developing social skills.

- Integration not segregation.

What I mean when I say it is a working video, is that it is a video with a purpose. It is a video meant to motivate, and to encourage people to achieve real outcomes of community integration. The intent is to 'help people with developmental disabilities break patterns of isolation and become more involved in their community'.

As we know, often individuals with disabilities may not experience the same opportunities for social/recreational activities as their peers without disabilities. Many individuals with disabilities live a partial and fragmented existence in which their 'friends' or connections are paid, in which their social opportunities are completely exclusionary. 'I Belong Out There' provides a humorous approach to a serious life aspect encouraging community members of all walks to end this isolation.

The video introduces the viewer to two Clydes: one is an active member of his community, the other is an isolated couch potato, who is seeing for the first time 'the Clyde he could be'. The active Clyde has a full-time job. This is important in the circle of Clyde's life. However, the role of leisure and social activities is also critical to enhance his quality of life. This active Clyde shows the isolated Clyde the possibilities for: recreation and fitness, hobbies, training, education, and volunteering.

The first anecdote is the ubiquitous bowling. I initially reacted with a stereotypical response of 'Oh no, bowling again'. However, it soon became clear that this bowling team was a little different. It was integrated, two members have disabilities, two do not. This activity set the tone for the video and the stories shared. All of the examples were integrated with individuals without disabilities: little league T-ball, theater, music, story telling, aerobics and weight lifting.

This video is a 24-min introduction to becoming an active member of one's community. It can be used to augment trainings or workshops in self-advocacy, community participation and person-centered planning. The video can be used to educate and increase the awareness of individuals with disabilities, family members, community members, and professionals.

A 16-page training packet accompanies the video. Information includes: Suggested Uses, Values Checklist, Social Skills, Friendships and Natural Supports, Volunteering and Helping Others, and Ideas for Training and Education, Recreation and Fitness, Hobbies and Crafts and finally a Resource List. The materials include brief narratives and checklists which are camera ready to be used as handouts.

I believe 'I Belong Out There' would be more 
effective if it was incorporated as an interactive exercise into an actual training, presentation or planning meeting. for example, 'I Belong Out There' would be introduced or 'set-up' by the trainer or respective presenter. The video would be followed by the presenter facilitating one or several of the checklist activities depending on the need and interest of the audience, a brainstorming activity, and/or a discussion. The total activity would then be summarized by the facilitator. Albeit, it may be functional as a stand-alone awareness vehicle which may influence attitudes of community members. I, personally, would tend to use it within a presentation/training format.

This is a useful video represented by the values of equal opportunity and inclusion. The quality of the video is somewhat fuzzy at times. In the process of duplication, the images have bled (due to color) in several of the scenes, lighting is a variable in other scenes. This is visually unfortu- nate, however, the content is not jeopardized. The video is available in closed and open captioning as well as audio narrative.

'I Belong Out There' contributes to the effort to develop holistic lives with individuals with developmental disabilities. It blows out a 'slice of the pie' by concentrating on an often neglected or challenging aspect of community inclusion, social/recreational outlets. Employment, in tandem with opportunities for social community connections, contributes to a well-rounded existence, one we all strive for. This video is a good start for individuals, with and without disabilities, to develop and engage in integrated social/ recreational activities.
Amy Armstrong Training Associate 